\title{
Nasal symptoms, epithelial injury and neurogenic inflammation in elite swimmers*
}

\author{
Brecht Steelant ${ }^{1}$, Valerie Hox ${ }^{1,2}$, Laura Van Gerven 1,2, Ellen Dilissen', \\ Emily Dekimpe ${ }^{2}$, Ahmad Kasran', Sven Aertgeerts', Vincent Van Belle \\ Koen Peers ${ }^{5}$, Lieven J. Dupont ${ }^{6,7}$, Peter W. Hellings ${ }^{1,2}$, Dominique M. Bullens ${ }^{8,9}$, \\ Sven F. Seys ${ }^{1,6}$ \\ ' Clinical Immunology, Department of Microbiology and Immunology, KU Leuven, Leuven, Belgium \\ ${ }^{2}$ Clinical Department of Otorhinolaryngology, Head and Neck Surgery, University Hospitals Leuven, Belgium \\ ${ }^{3}$ Academic Center for General Practice, KU Leuven, Leuven, Belgium \\ ${ }^{4}$ Flemish Swimming Federation, Merelbeke, Belgium \\ ${ }^{5}$ Sport Medical Advice Centre, University Hospitals Leuven, Belgium \\ ${ }^{6}$ Laboratory of Pneumology, KU Leuven, Leuven, Belgium \\ ${ }^{7}$ Clinical Department of Pneumology, University Hospitals Leuven, Belgium \\ ${ }^{8}$ Pediatric Immunology, Department of Microbiology and Immunology, KU Leuven, Leuven, Belgium \\ ${ }^{9}$ Clinical Department of Pediatrics, University Hospitals Leuven, Belgium
}

Rhinology 56: 3, 279-287, 2018

https://doi.org/10.4193/Rhin17.167

*Received for publication:

July 27, 2017 Accepted: February 12, 2018

Background: A high burden of lower airway symptoms is found in elite swimmers. To what extent elite swimmers suffer from upper airway symptoms and how these associate with nasal inflammation is less clear. We here aimed to evaluate upper airway symptoms and nasal inflammation in elite athletes.

Methodology: Elite swimmers, indoor athletes and age-matched controls were recruited. Upper airway symptoms were assessed by sino-nasal outcome test (SNOT)-22 questionnaire. Visual Analogue score (VAS) for nasal symptoms as well as neurogenic and inflammatory mediators in nasal fluid were assessed at baseline, immediately and 24-hours after sport-specific training. The effect of hypochlorite on nasal epithelial cells was evaluated in vitro.

Results: Baseline SNOT-22 and VAS for nasal itch and impaired smell were significantly higher in swimmers compared to controls. Nasal substance $P$ and uric acid levels were increased in elite swimmers 24-hours after swimming compared to baseline. In elite swimmers, uric acid levels 24-hours post-exercise correlated with baseline SNOT-22. As increased symptoms and inflammation were found in swimmers but not in indoor athletes, we hypothesized that hypochlorite exposure might be the underlying mechanism. In vitro, the highest dose of hypochlorite decreased nasal epithelial cell integrity and induced release of uric acid.

Conclusion: Upper airway symptoms are frequently reported in elite swimmers. Intensive swimming resulted in a delayed increase of epithelial injury and neurogenic inflammation.

Key words: Elite athletes, Substance P, Uric acid, hypochlorite, exercise

\section{Introduction}

Elite athletes perform intensive physical exercise on a daily basis. There is an increased awareness that intensive training is associated with a higher risk to develop exercise-induced bronchoconstriction or asthma ${ }^{(1,2)}$. Upper airway symptoms might be present in an even higher proportion of athletes; however, this has been less studied ${ }^{(3-6)}$. A 12-year survey of 650 Italian Olympic athletes showed the presence of rhinitis in $26 \%$ of cases ${ }^{(7)}$. More recently, $36 \%$ of elite athletes had physician-diagnosed rhinitis (8). In elite swimmers, it has been shown that a training session induced nasal symptoms and reduced Rhinitis Quality of Life Questionnaire score ${ }^{(9)}$. During exercise, the airflow increases meaning that the airways of athletes are exposed to increased passage of air. Hence, more pollutants, allergens as well as ir- 
ritants can enter the airways ${ }^{(10)}$. These factors may lead to airway epithelial cell injury with the development of airway symptoms related to asthma and/or rhinitis ${ }^{(11)}$. Indeed, we previously demonstrated an increase in damage associated molecular patterns and pro-inflammatory cytokines in sputum of elite swimmers ${ }^{(12)}$

To our knowledge, little research has been conducted to evaluate the effect of intensive sports performance on upper airway symptoms in elite athletes ${ }^{(13-17)}$. Consequently, we evaluated nasal symptoms using sino-nasal outcome test-22

(SNOT-22) questionnaire ${ }^{(18)}$ and visual analogue scales (VAS) in elite swimmers, indoor athletes and age-matched controls. We next evaluated whether sport-specific training, i.e. 90 minutes swimming for elite swimmers and age-matched controls and a 90 minutes indoor sport-training for indoor athletes, resulted in more nasal symptoms. Lastly, we evaluated markers for neurogenic inflammation and epithelial damage in nasal secretions of elite athletes.

\section{Subjects and methods}

Subjects

Competitive swimmers $(n=38)$ and indoor athletes $(n=13$; i.e. volleyball or basketball players) were recruited among Belgian teams performing at national and international level. Age-matched controls ( $n=15$ ) were capable of swimming for 90 minutes and did not perform sports for more than 4 hours on a weekly basis. Details on the study protocol are published elsewhere ${ }^{(12)}$. Swimmers, indoor athletes and controls were initially evaluated for exercise-induced bronchoconstriction and inflammation of the lower airways ${ }^{(12)}$. All study participants were evaluated before, immediately after and 24-hours after an intensive training session, i.e. 90 minutes indoor swimming for swimmers and agematched controls and an indoor sport-specific training session (either volleyball or basketball) for indoor athletes. All swimmers trained in chlorinated pools, in identical circumstances as reported previously ${ }^{(12)}$. Competitive swimmers were studied outside pollen season as this might affect study outcome. More information on the training protocol has been reported previously ${ }^{(12)}$. Baseline characteristics are depicted in Table 1.

The study was approved by the institutional review board and is registered at clinicaltrials.gov (NCT01942096). All subjects gave written informed consent before participation in this study.

\section{Clinical evaluation}

Baseline symptoms of upper airways were assessed by Dutch Total Sino-Nasal Outcome Test (SNOT-22) questionnaire by Piccirillo et al. ${ }^{(19,20)}$. SNOT-22 was divided in five subgroups; i.e. nasal, paranasal and sleep-related symptoms, and social and emotional impairment ${ }^{(21)}$. VAS scores for runny nose; blocked nose; facial pain; impaired smell; itchy nose; postnasal drip; sneezing; and irritation in the nose, were assessed at baseline, immedia-
Table 1. Subject characteristics.

\begin{tabular}{|lcccc|}
\hline & $\begin{array}{c}\text { Swim- } \\
\text { mers }\end{array}$ & $\begin{array}{c}\text { Indoor } \\
\text { athletes }\end{array}$ & Controls & P value \\
\hline Number $(\mathrm{n}=)$ & 38 & 13 & 15 & \\
\hline Age (years) & $17.0 \pm 2.4$ & $18.5 \pm 1.8$ & $19.2 \pm 3.6$ & .18 \\
\hline Gender (M / F) & $23 / 15$ & $8 / 5$ & $7 / 8$ & .05 \\
\hline Atopy (n=) & $13(34 \%)$ & $3(23 \%)$ & $4(27 \%)$ & .71 \\
\hline Training (years) & $9.5 \pm 2.3$ & $9.5 \pm 2.4$ & n.a. & .97 \\
\hline Training / week (hours) & $15.7 \pm 3.6$ & $14.8 \pm 1.0$ & $<4.0$ & .49 \\
\hline $\begin{array}{l}\text { Previous physician- } \\
\text { diagnosed asthma }\end{array}$ & $16(42 \%)$ & $1(8 \%)$ & $1(7 \%)$ & .007 \\
\hline $\begin{array}{l}\text { Inhaled } \beta 2 \text {-agonists } \\
\text { only }\end{array}$ & $2(5 \%)$ & 0 & 0 & .47 \\
\hline \begin{tabular}{l} 
Inhaled corticosteroids \\
\hline Nasal corticosteroids
\end{tabular} & $8(21 \%)$ & 0 & 0 & .03 \\
\hline
\end{tabular}

Atopy was defined if skin prick test was positive for at least one aeroallergen.

tely after and 24-hours after the intensive training.

\section{Nasal fluid collection}

To collect nasal fluid, a nasal sponge (Ivalon Surgical products, San Diego, CA, USA: Post-Op Sinus Pack, K9) was weighed and inserted into one of the nostrils for 5 minutes at three different time points: baseline, immediately after and 24 hours after sport-specific training. After 5 minutes, the sponge was removed and weighed again. A volume of saline was added depending on the weight of the collected sponge ( $1 / 5$ dilution). The sponge was then squeezed and centrifuged at $1500 \mathrm{~g}$ at $4^{\circ} \mathrm{C}$ for $5 \mathrm{mi}-$ nutes. Supernatant was stored at $-20^{\circ} \mathrm{C}$ upon analysis.

\section{Peak Nasal Inspiratory Flow (PNIF)}

Nasal obstruction was evaluated using the peak nasal inspiratory flow (PNIF) device (Cement Clarkx International, Essex, UK) and was measured as previously described ${ }^{(22)}$.

\section{Analysis of mediators in nasal fluid}

Due to the small volume of nasal fluid that was collected, IL-1 $\beta$, IL- 6 and TNF were selected for analysis since these have been shown to be significantly increased in sputum after swimming, whereas no changes in T cell cytokines were observed ${ }^{(12)}$. IL-1 $\beta$, IL-6 and tumor necrosis factor (TNF) levels were determined in nasal fluid by Cytometric Bead Array according to the manufacturers' protocol (BD biosciences, Erembodegem, Belgium). The levels of neuronal proteins Substance $P(S P)$ and Nerve growth factor (NGF) were determined in the nasal fluid of elite swimmers as described previously ${ }^{(23)}$. 
Uric acid levels were quantified in the nasal fluid of elite swimmers by Amplex Red Uric Acid/Uricase Assay kit from Life Technologies (Carlsbad, CA, USA) according to the manufacturers' protocol.

Isolation of primary nasal epithelial cells and trans-epithelial electrical resistance measurements

Inferior turbinates of healthy, non-allergic, non-asthmatic, nonsmoking control subjects were used for isolation of nasal epithelial cells (NECs). A highly purified NEC population was obtained as reported previously ${ }^{(24)}$. Freshly isolated NECs were seeded on $0.4 \mu \mathrm{m} 0.33 \mathrm{~cm}^{2}$ transwell inserts (Greiner Bio One, Vilvoorde, Belgium) at a density of 100.000 cells/transwell. Culture medium DMEM-F12, supplemented with $100 \mathrm{U} / \mathrm{ml}$ penicillin, $100 \mu \mathrm{g} / \mathrm{ml}$ streptomycin, and 2\% Ultroser G (Pall Life Sciences, Hoegaarden, Belgium) was refreshed every other day. Once epithelial cells reached complete confluence, the apical culture medium was removed to allow further cell differentiation in air-liquid interface. At day 21 in air-liquid interface, epithelial integrity was evaluated by trans-epithelial resistance (TER) measurements using an EVOM/Endohm (WPI Inc, Sarasota, FL, USA).

Effect of hypochlorite on primary nasal epithelial cells integrity and viability

NECs were stimulated at day 21 by adding different concentrations of hypochlorite (ClO-), a disinfectant with irritant capacities ${ }^{(25)}$ : the swimming pool concentration $0.0003 \%$ and 10 and 100 times higher $(0.003 \%$ and $0.03 \%)$ concentration. TER was measured in function of time and is expressed as relative change to baseline values. To investigate hypochlorite-induced cytotoxicity, NECs were stimulated with hypochlorite for 24 hours and cell viability was analyzed via MTT (3-(4,5- dimethylthiazol-2-yl)-2,5diphenyltetrazolium bromide) assays, as described previously (26).

\section{Statistical analysis}

Data were analyzed using Graphpad Prism 5 (La Jolla, CA, USA). One-way ANOVA or Kruskal-Wallis test with post-hoc analysis (Holm-Sidak's or Dunn's, respectively) was used to compare multiple groups. Normality was analyzed prior to group analyses by Shapiro-Wilk test. Categorical values were compared using the Fisher exact test. Pearson's r or Spearman's $\rho$ were used to determine correlations. Values were considered significantly different when $\mathrm{p}<0.05$.

\section{Results}

Subjects

Baseline characteristics are presented in Table 1. Atopy was equally distributed amongst the three groups $(p=0.86)$. Previously physician diagnosed asthma (based on questionnaires) was significantly higher in elite swimmers $(n=16)$ compared to

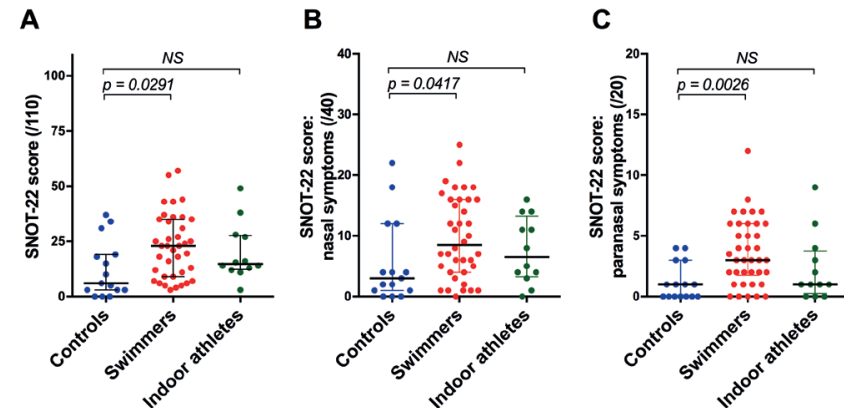

Figure 1. Baseline SNOT-22 scores in controls, elite swimmers and indoor athletes. A) Total SNOT-22 score is significantly higher in elite swimmers compared to controls. B), C) Sub-analysis of SNOT-22 scores showed more nasal- and paranasal symptoms in elite swimmers compared to controls. Data presented as median and interquartile range. KruskalWallis test with post-hoc analysis, NS = not significant.

indoor athletes and controls ( $p<0.01$; Table 1). Ten swimmers were using anti-asthmatic medication (inhaled corticosteroids: $\mathrm{n}=8$ and inhaled short-acting $\beta 2$-agonists: $\mathrm{n}=2$ ). Four swimmers were using nasal corticosteroids daily.

Elite athletes reported more baseline upper airway symptoms We first investigated whether elite athletes had more nasal symptoms at baseline compared to controls. Therefore, upper airway symptoms were evaluated using SNOT-22 questionnaire and VAS scores. Elite swimmers scored significantly higher on the SNOT-22 questionnaire compared to age-matched controls $(p<0.05$, Figure $1 A)$. Neither asthma nor atopy was associated with increased SNOT-22 scores in elite swimmers. A sub-analysis of baseline SNOT-22 scores showed significantly higher scores for nasal $(p<0.05$, Figure 1B) and paranasal symptoms $(p<0.01$, Figure $1 C$ ) in elite swimmers compared to age-matched controls, which was not found in indoor athletes compared to agematched controls (Figure 1B-C).

In line with the SNOT-22 scores for nasal and paranasal symptoms, baseline VAS for nasal itch $(p<0.05$, Figure $2 A)$ and impaired smell $(P<0.05$, Figure $2 B$ ) was significantly higher in elite swimmers but not in indoor athletes compared to controls. No significant differences in symptoms were found between elite swimmers and controls for baseline nasal obstruction, rhinorrhoea (Figure 2C, D) and sneezing (0.3 (0 - 1.8) vs. 0.2 (0 2.1), $p=0.92$, data not shown).

In contrast, sport-specific training significantly increased symptoms of rhinorrhoea in indoor athletes immediately after training, which decreased again after 24 -hours to baseline values ( $p<0.01$, Figure $3 \mathrm{~A}$ ). Rhinorrhoea symptoms were not affected by sport-specific training in elite swimmers. However, VAS scores for rhinorrhoea in age-matched controls decreased instantly after indoor swimming ( $p<0.05$, Figure $3 A$ ). Interestingly, VAS for nasal obstruction was significantly lower in elite 
A

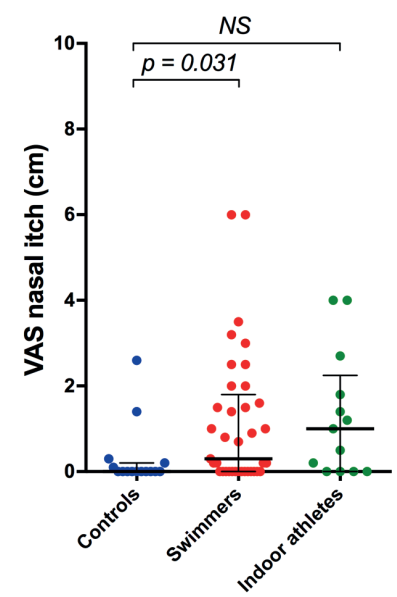

B

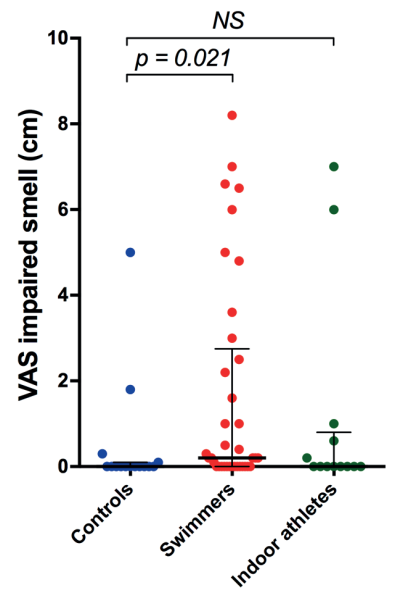

C

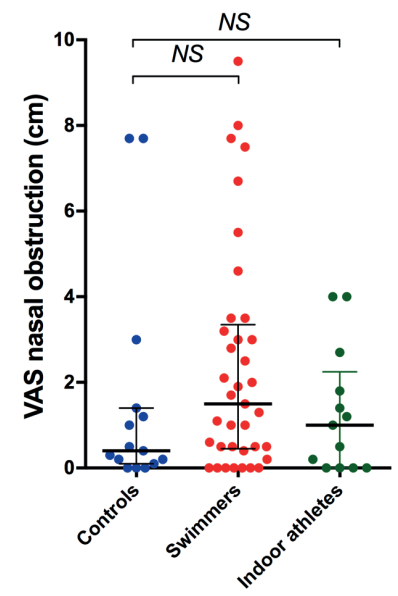

D

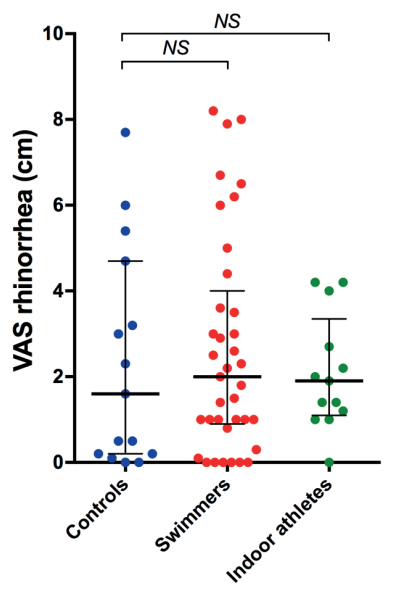

Figure 2. Nasal symptoms at baseline in controls, elite swimmers and indoor athletes. A) VAS for nasal itch and B) VAS for smell impairment are significantly higher in elite swimmers compared to controls. C), D) VAS for nasal obstruction and rhinorrhea did not differ between the groups. Data presented as median and interquartile range. Kruskal-Wallis test with post-hoc analysis. NS = not significant

A
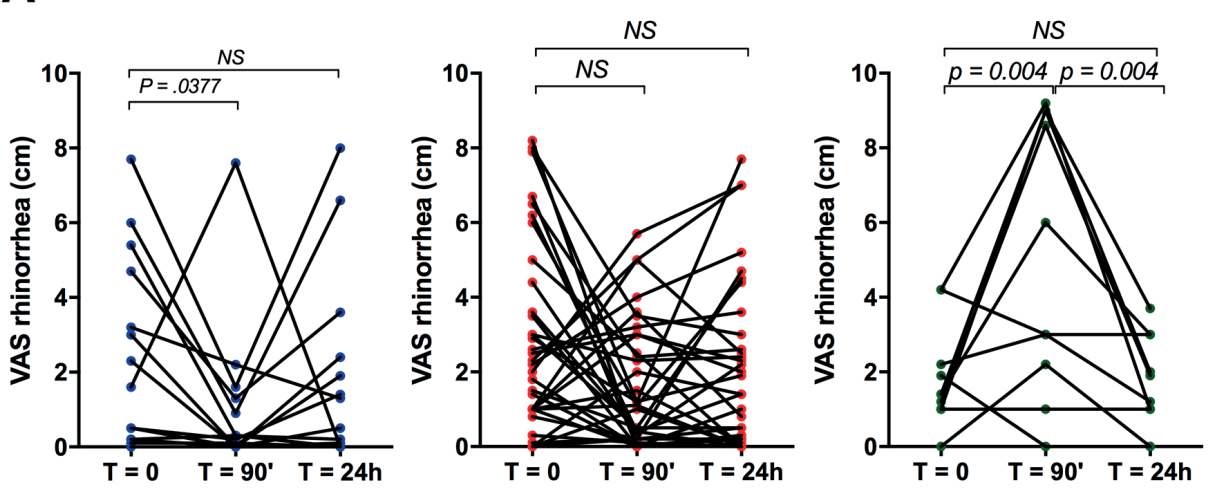

B

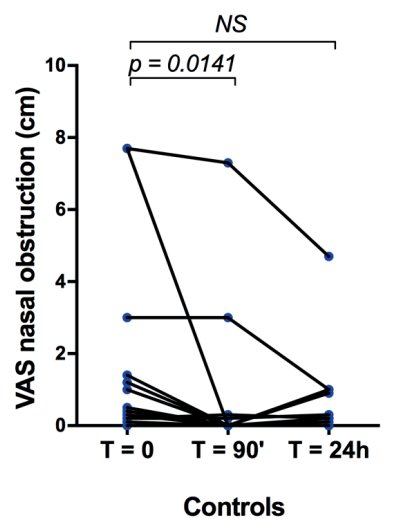

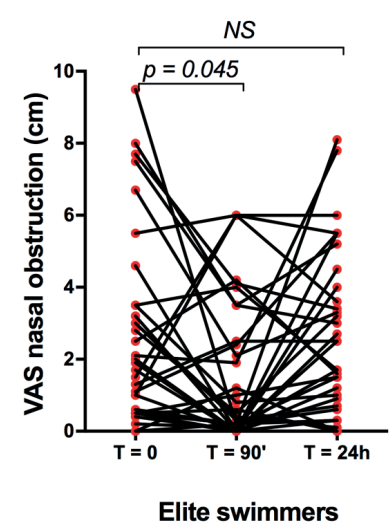

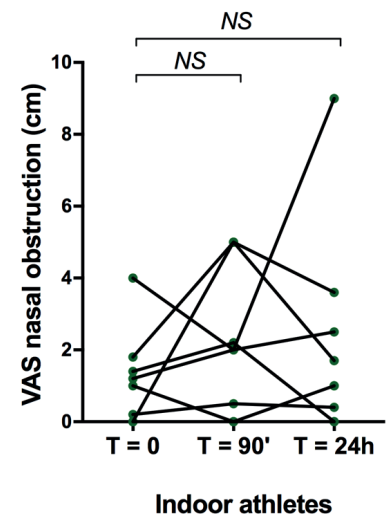

Figure 3. Sport-specific training and the development of nasal symptoms. A) Sport specific training induced rhinorrhoea in indoor athletes but not in elite swimmers. B) Nasal obstruction decreased after 90 minutes swimming in controls and elite swimmers but not in indoor athletes. Friedman test, NS = not significant.

swimmers and age-matched controls immediately after indoor swimming compared to baseline (both $p<0.05$, Figure 3B),

whereas no changes in VAS for nasal obstruction were observed in indoor athletes (Figure 3B).

PNIF measurements did not significantly differ among three groups (Supplementary Table E1) nor altered immediately after 
A

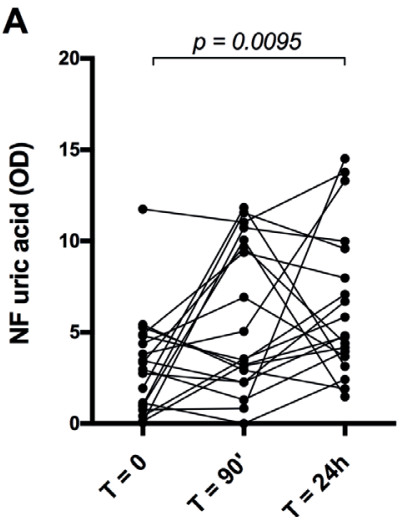

C

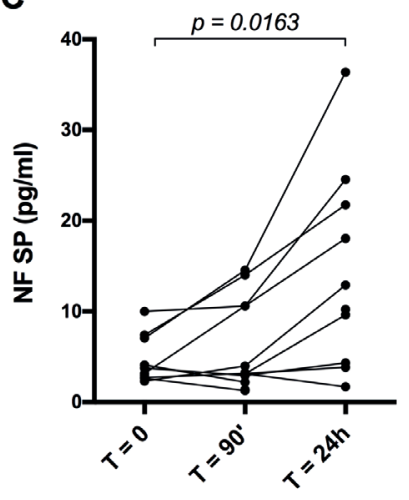

B

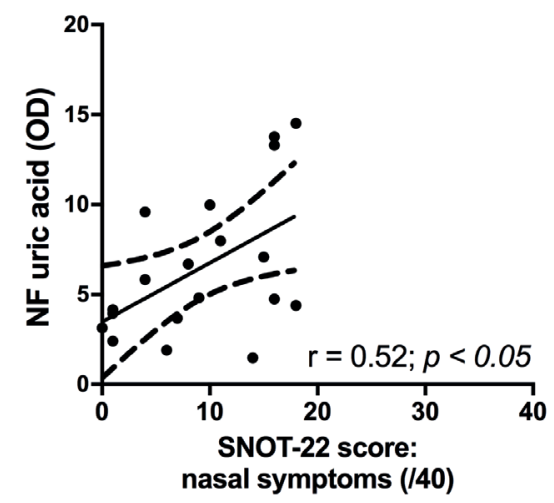

D

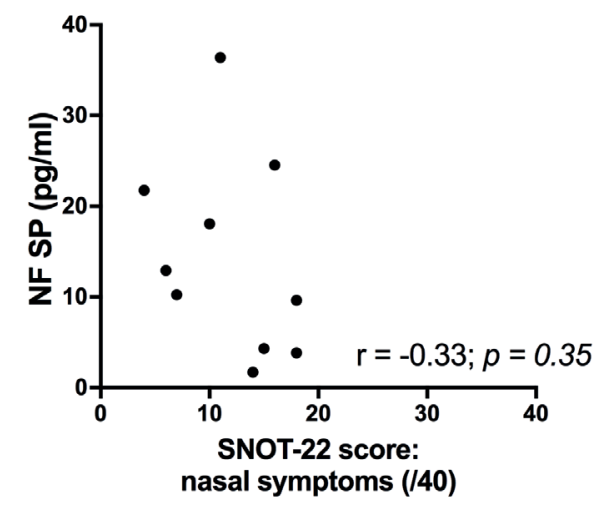

Figure 4. Uric acid and substance P levels in nasal secretions of elite swimmers at baseline, 90 minutes and 24-hours after a 90 minutes swimming session. A) Uric acid levels were significantly increased 24-hours after an intensive swimming session ( $n=19)$. B) Correlation between uric acid levels after 24 hours and baseline SNOT-22 score for nasal symptoms. C) Substance P levels were significantly increased 24-hours post sport ( $\mathrm{n}=10$ ). D) No correlation between substance $P$ and baseline SNOT-22 score for nasal symptoms. NF: nasal fluid.

or 24-hours after sport-specific training (Supplementary Figure E1).

Swimming induced nasal epithelial injury and neurogenic inflammation

As elite swimmers were the group with most baseline complaints, we investigated whether swimming in an indoor chlorinated swimming pool could result in injury of the nasal epithelium. For this, a danger associated molecular pattern molecule, i.e. uric acid, was measured in the nasal fluid of elite swimmers at baseline, immediately after and 24-hours after intensive training ( $n=19)$. Uric acid levels were significantly elevated 24-hours after intensive training compared to baseline levels (Figure $4 A, p<0.05)$. No increased levels of uric acid levels after sportspecific training were found in indoor athletes and age-matched controls (data not shown). Moreover, in elite swimmers, uric acid levels measured at 24-hours correlated with baseline SNOT-22 subscore for nasal symptoms ( $r=.52, p<0.05$, Figure 4B). In addition, SP levels were significantly elevated 24-hours after an intense swimming session in elite swimmers $(n=10)$ (Figure
$4 C, p<0.05)$, while NGF levels remained unaltered $(n=11)(26$ (22-49) vs. 25 (22-32) pg/ml, p=0.38). No correlation was found between SP levels and baseline SNOT-22 subscore for nasal symptoms in elite swimmers (Figure 4D). Nasal fluid IL1 $\beta$ and IL-6 levels were unchanged at both time points after swimming compared to baseline (data not shown), whereas TNF could not be detected.

Ultimately, as exposure to chlorinated pools might explain why swimmers have more complaints, we studied if hypochlorite (ClO-) could disrupt nasal epithelial cell integrity in vitro. Therefore, primary NECs isolated from healthy controls were stimulated with different concentrations $(0.03-0.003-$ $0.0003 \%$ ) of $\mathrm{ClO}$ - and epithelial integrity was evaluated in time. The swimming pool concentration of $\mathrm{ClO}$-, i.e. $0.0003 \%$ did not affect TER, while increased doses of CIO- $(0.03 \%)$ decreased TER after 24-hours (Figure $5 \mathrm{~A}, \mathrm{p}<0.05)$. The effect of $\mathrm{ClO}-(0.03 \%)$ was linked with decreased cell viability, as measured by MTT assay (Figure 5B, p <0.05). In addition, a significant increase in uric acid was measured in the supernatant after stimulation with $\mathrm{ClO}$ $(0.0003 \%)$ (Figure $5 \mathrm{C}, \mathrm{p}<0.05)$. No significant increase in uric acid 
A

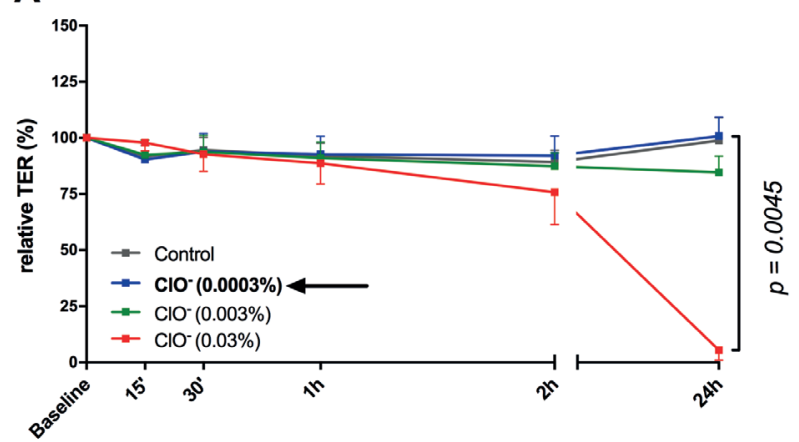

B

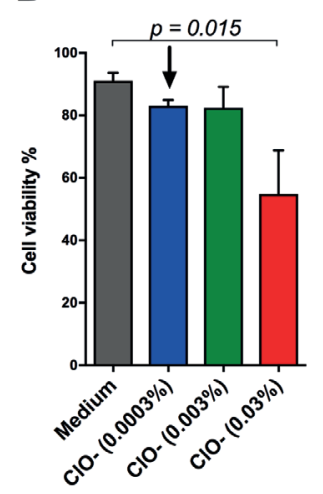

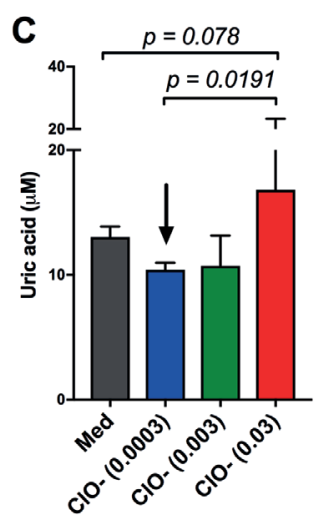

Figure 5. Effect of hypochlorite on primary nasal epithelial cells derived from healthy controls ( $n=6)$. A) Effect of 24-hour stimulation with different concentrations of hypochlorite (ClO-) on nasal epithelial cell (NEC) integrity in vitro $(n=6)$. B) Cell viability measured with MTT assay. C) Uric acid release measured in the supernatant of NECs stimulated for 24 hours with ClO-. Kruskal-Wallis with post-hoc analysis. Black arrow indicates swimming pool concentration of $\mathrm{ClO}$.

was measured compared to medium control.

\section{Discussion}

Rhinitis symptoms, either allergic or non-allergic, are frequently observed in elite athletes and are the highest in elite swimmers ${ }^{(4-6)}$. In the latter case, exposure to chlorination products in swimming pools seems to play a relevant role, since it has been shown that these products can exacerbate pre-existing allergic nasal obstruction or induce non-specific irritation of the nasal mucosa ${ }^{(27)}$. In this study, we explored nasal symptoms and inflammation in elite swimmers and compared these to indoor athletes and age-matched control subjects. To our knowledge, only a limited number of studies has focused on the effect of a training session on upper airway symptoms and inflammation in athletes ${ }^{(13)}$.

SNOT-22 scores were significantly higher at baseline in elite swimmers compared to age-matched controls, independent of the atopic or asthmatic status of the subject in our cohort. We analysed SNOT-22 subscores for nasal, paranasal, sleep-related, social and emotional factors ${ }^{(28)}$. SNOT-22 subscores for nasal

and paranasal factors and not sleep-related, social or emotional factors were significantly higher in elite swimmers compared to controls. This confirms in a standardized way the high prevalence of elite swimmers reporting upper airway symptoms as the most disturbing factor during the training season ${ }^{(9)}$. Interestingly, usage of a nasal clip for 30 days, significantly reduced nasal VAS scores for nasal symptoms ${ }^{(5)}$.

Moreover, at baseline, elite swimmers had an "impaired smell" and "itchy nose" (assessed by VAS) compared to age-matched controls and to a lesser extend to indoor athletes. The finding of "impaired smell" is in line with a previous report by Ottaviano et al. who tested the olfactory threshold for $n$-butanol in a population of competitive swimmers and controls ${ }^{(29)}$. Higher n-butanol thresholds were found in competitive swimmers, which is one of the most sensitive signs of toxic epithelial damage. We are aware of the subjective measurement of "smell impairment" by VAS in this study, but it at least could lead to more in-depth studies objectively studying smell in elite swimmers. The higher baseline "nasal itch" found in elite swimmers compared to age-matched controls might be partly explained by chronic chlorine-induced activation of the transient receptor potential channel (TRP)A1 ${ }^{(30)}$. Indeed, TRPA 1 is a key channel responsible for the transmission of itch sensation ${ }^{(31)}$. Twenty-four hours after an intensive indoor swimming session, we found significantly increased SP levels in nasal fluid of elite swimmers, which points towards TRPA 1 activation and the release of neuropeptide 24-hours after intensive training. In mice, we have previously shown that hypochlorite can stimulate TRPA1, resulting in the local release of SP, which could bind its neurokinin receptor 1 and the subsequent induction of airway hyperreactivity ${ }^{\left({ }^{30}\right)}$. We therefore hypothesize that exposure to chlorination products can stimulate the release of SP in the upper airways by activating TRPA1, which induces symptoms and more specifically "nasal itch" by binding to the neurokinin receptor 1 , found on local mast cells and endothelial cells ${ }^{(32)}$. NGF, another neuro-mediator, was not altered after intensive swimming.

Rhinorrhoea was increased in indoor athletes immediately after sport-specific training compared to baseline, but not in elite swimmers and controls. In our perception, the lack of increased rhinorrhoea symptoms in elite swimmers might be explained by the timing of the scoring and/or the high baseline levels in swimmers and, to our surprise, also in age-matched controls. Indeed, nasal symptoms were scored immediately after leaving the water, making the perception of rhinorrhoea hard to distinguish from pool water dripping of the face. Moreover, we have to stress that indoor athletes performed a sport-specific training, which was obviously not in a swimming pool.

Nasal obstruction was lower after 90 minutes swimming in elite swimmers and age-matched controls compared to baseline. Forsyth and co-workers described already in 1983 a decrease in nasal resistance, following stimulation of the sympathetic 
nervous system during physical activity ${ }^{(33)}$. However, in this study the subjective drop in nasal airflow, was not accompanied by a significant decrease in PNIF values. Despite the induction of some nasal symptoms in elite swimmers and indoor athletes by sport-specific training, nasal patency (PNIF) and the volume of nasal secretions did not seem to decrease or increase respectively after sport-specific training, which is in line with previous publications $(5,9,17,29,34)$.

Lastly, we have investigated whether exposure to chlorination products, used in swimming pools, is associated with nasal epithelial cell injury and induction of nasal inflammation. Increased sputum levels of danger associated molecular patterns such as High Mobility Group Box-1 and uric acid, and increased serum levels of Clara cell protein-16 are found in elite swimmers, pointing to inflammation and epithelial cell damage in the lower airways ${ }^{(12,35)}$. These results support the hypothesis that epithelial injury is contributing to exercise induced bronchoconstriction in elite swimmers. Indeed, Carbonelle and colleagues demonstrated a greater epithelial permeability in swimmers training in chlorinated pools compared to those training in copper-sliver pools ${ }^{(35)}$. In order to translate these results to the upper airways, we measured uric acid in nasal fluid from elite swimmers. Elite swimmers showed a significant increase in nasal uric acid 24 hours after sport-specific training compared to baseline values. Importantly, uric acid levels after 24 hours correlated with baseline SNOT-22 subscores for nasal symptoms, suggesting that exposure to chlorinated swimming pools can cause injury of the upper airway epithelium and therefore facilitate the induction of nasal symptoms.

Moreover, we found a dose dependent increase in uric acid release from NECs after stimulation with hypochlorite in vitro. However, swimming pool concentrations did not induce significant changes. This was accompanied by cell death at the highest concentration and loss of epithelial integrity. We hypothesize however that prolonged exposure to these (higher) concentrations of hypochlorite might be able to induce some signs of epithelial injury. Alternatively, sudden increase in by-products in case of an over representation to chlorine products might therefore have harmful effects ${ }^{(36)}$

\section{Conclusion}

In conclusion, upper airway symptoms, and more specifically baseline nasal and paranasal symptoms, were found in a significant proportion of elite swimmers. Nasal symptoms correlated with uric acid levels in nasal secretions 24-hours post-exercise, which suggest that epithelial injury contributes to the development of nasal symptoms in these swimmers. Neurogenic inflammation might contribute to increased nasal symptoms, especially to increased itch in elite swimmers. Evaluation of treatment strategies that interfere with these pathways in elite swimmers are now needed to confirm the link between epithelial injury, neurogenic inflammation and upper airway symptoms.

\section{Acknowledgement}

The study was approved by the institutional review board and is registered at clinicaltrials.gov (NCT01942096). All subjects gave written informed consent before participation in this study. The author's laboratories are supported by grants from the Climbing for Life research fund, the Belgian Federal Government (IUAP P7/30) and the research council of the KU Leuven (GOA 2009/07 and 14/011). P.W.H., L.D. and D.MA.B. are recipients of a senior researcher fellowship from the Fund of Scientific Research (FWO), Flanders, Belgium.

\section{Authorship contribution}

S. A., V. V. B., K. P., L. J. D., D. M. B. and S. F. S. participated in the study design. B. S., V. H., A. K. and S. F. S. participated in the study conduct. B. S., V. H., L. V. G., E. D., E.D. and S. F. S. participated in data collection and analysis. B. S., P. W. H., D. M. B. and S. F. S. participated in data interpretation. L. J. D. and D. M. B. participated in drafting the article. All authors revised and approved the final version of the article. S. F. S., D. M. B. and B. S. take responsibility for the integrity of the data analysis.

\section{Conflict of interest}

The authors have no conflict of interest.

\section{References}

1. Boulet LP. Cough and upper airway disorders in elite athletes: a critical review. $\mathrm{Br}$ Sports Med. 2012;46(6):417-21.

2. Carlsen $\mathrm{KH}$, Anderson SD, Bjermer L, Bonini S, Brusasco V, Canonica W, et al. Exerciseinduced asthma, respiratory and allergic disorders in elite athletes: epidemiology, mechanisms and diagnosis: part I of the report from the Joint Task Force of the European Respiratory Society (ERS) and the European Academy of Allergy and Clinical Immunology (EAACI) in cooperation with GA2LEN. Allergy. 2008;63(4):387-403.

3. Schwartz LB, Delgado L, Craig T, Bonin
S, Carlsen KH, Casale TB, et al. Exerciseinduced hypersensitivity syndromes in recreational and competitive athletes: a PRACTALL consensus report (what the general practitioner should know about sports and allergy). Allergy. 2008;63(8):953-61.

4. Belda J, Ricart S, Casan P, Giner J, BellidoCasado J, Torrejon M, et al. Airway inflammation in the elite athlete and type of sport. Br J Sports Med. 2008;42(4):244-8; discussion 8-9.

5. Gelardi M, Ventura MT, Fiorella R, Fiorella ML, Russo C, Candreva T, et al. Allergic and non-allergic rhinitis in swimmers: clinical and cytological aspects. Br J Sports Med.
2012;46(1):54-8

6. Bonini S, Bonini M, Bousquet J, Brusasco V, Canonica GW, Carlsen KH, et al. Rhinitis and asthma in athletes: an ARIA document in collaboration with GA2LEN. Allergy. 2006;61(6):681-92.

7. Bonini M, Gramiccioni C, Fioretti D, Ruckert B, Rinaldi M, Akdis C, et al. Asthma, allergy and the Olympics: a 12-year survey in elite athletes. Curr Opin Allergy Clin Immunol. 2015;15(2):184-92

8. Couto M, Stang J, Horta L, Stensrud T, Severo M, Mowinckel $P$, et al. Two distinct phenotypes of asthma in elite athletes identified by latent class analysis. J Asthma. 
2015;52(9):897-904

9. Bougault V, Turmel J, Boulet LP. Effect of intense swimming training on rhinitis in high-level competitive swimmers. Clin Exp Allergy. 2010;40(8):1238-46.

10. Kippelen P, Fitch KD, Anderson SD, Bougault $V$, Boulet LP, Rundell KW, et al. Respiratory health of elite athletes - preventing airway injury: a critical review. Br J Sports Med. 2012:46(7):471-6.

11. Hallstrand TS, Moody MW, Wurfel MM, Schwartz LB, Henderson WR, Jr., Aitken ML. Inflammatory basis of exercise-induced bronchoconstriction. Am J Respir Crit Care Med. 2005;172(6):679-86.

12. Seys SF, Hox V, Van Gerven L, Dilissen E, Marijsse G, Peeters E, et al. Damageassociated molecular pattern and innate cytokine release in the airways of competitive swimmers. Allergy. 2015;70(2):187-94.

13. Clearie KL, Vaidyanathan S, Williamson PA, Goudie A, Short P, Schembri S, et al. Effects of chlorine and exercise on the unified airway in adolescent elite Scottish swimmers. Allergy. 2010;65(2):269-73.

14. Clearie KL, Williamson $P A$, Vaidyanathan $S$, Short P, Goudie A, Burns P, et al. Disconnect between standardized field-based testing and mannitol challenge in Scottish elite swimmers. Clin Exp Allergy. 2010;40(5):7317.

15. Kurowski M, Jurczyk J, Krysztofiak $H$, Kowalski ML. Exercise-induced respiratory symptoms and allergy in elite athletes: Allergy and Asthma in Polish Olympic Athletes (A(2)POLO) project within GA(2) LEN initiative. Clin Respir J. 2016;10(2):231-8.

16. Katelaris CH, Carrozzi FM, Burke TV, Byth K. Patterns of allergic reactivity and disease in Olympic athletes. Clin J Sport Med. 2006;16(5):401-5.

17. Alves A, Martins C, Delgado L, Fonseca J, Moreira A. Exercise-induced rhinitis in competitive swimmers. Am J Rhinol Allergy. 2010;24(5):e114-7.

18. Kennedy JL, Hubbard MA, Huyett P, Patrie JT, Borish L, Payne SC. Sino-nasal outcome test (SNOT-22): a predictor of postsurgical improvement in patients with chronic sinusitis. Ann Allergy Asthma Immunol. 2013;111(4):246-51 e2.

19. Piccirillo JF, Merritt MG, Jr., Richards ML. Psychometric and clinimetric validity of the 20-Item Sino-Nasal Outcome Test (SNOT-20). Otolaryngol Head Neck Surg.
2002;126(1):41-7.

20. Rudmik L, Hopkins C, Peters A, Smith TL, Schlosser RJ, Soler ZM. Patient-reported outcome measures for adult chronic rhinosinusitis: A systematic review and quality assessment. J Allergy Clin Immunol. 2015;136(6):1532-40 e 1-2.

21. Hopkins C, Gillett S, Slack R, Lund VJ, Browne JP. Psychometric validity of the 22-item Sinonasal Outcome Test. Clin Otolaryngol. 2009;34(5):447-54.

22. Van Gerven L, Boeckxstaens G, Jorissen M Fokkens W, Hellings PW. Short-time cold dry air exposure: a useful diagnostic tool for nasal hyperresponsiveness. Laryngoscope. 2012;122(12):2615-20

23. Van Gerven L, Alpizar YA, Wouters MM, Hox $V$, Hauben $E$, Jorissen $M$, et al. Capsaicin treatment reduces nasal hyperreactivity and transient receptor potential cation channel subfamily V, receptor 1 (TRPV1) overexpression in patients with idiopathic rhinitis. J Allergy Clin Immunol. 2014;133(5):1332-9, 9 e1-3.

24. Steelant B, Farre R, Wawrzyniak P, Belmans J, Dekimpe E, Vanheel $H$, et al. Impaired barrier function in patients with house dust mite-induced allergic rhinitis is accompanied by decreased occludin and zonula occludens-1 expression. J Allergy Clin Immunol. 2016;137(4):1043-53 e1-5.

25. Bernard A. Chlorination products: emerging links with allergic diseases. Curr Med Chem. 2007;14(16):1771-82.

26. Maes W, Rosas GG, Verbinnen B, Boon L, De Vleeschouwer S, Ceuppens JL, et al. DC vaccination with anti-CD25 treatment leads to long-term immunity against experimental glioma. Neuro Oncol. 2009;11(5):529-42.

27. Shusterman D, Murphy MA, Balmes J. Influence of age, gender, and allergy status on nasal reactivity to inhaled chlorine. Inhal Toxicol. 2003;15(12):1179-89.

28. Hox V, Delrue S, Scheers H, Adams E, Keirsbilck $S$, Jorissen $M$, et al. Negative impact of occupational exposure on surgical outcome in patients with rhinosinusitis. Allergy. 2012;67(4):560-5.

29. Ottaviano G, Staffieri A, Stritoni P, Ermolao A, Coles S, Zaccaria M, et al. Nasal dysfunction induced by chlorinate water in competitive swimmers. Rhinology. 2012;50(3):294-8.

30. Hox V, Vanoirbeek JA, Alpizar YA, Voedisch S, Callebaut I, Bobic S, et al. Crucial role of transient receptor potential ankyrin 1 and mast cells in induction of nonallergic airway hyperreactivity in mice. Am J Respir Crit Care Med. 2013;187(5):486-93.

31. Wilson SR, Gerhold KA, Bifolck-Fisher A, Liu Q, Patel KN, Dong X, et al. TRPA1 is required for histamine-independent, Mas-related $G$ protein-coupled receptor-mediated itch. Nat Neurosci. 2011;14(5):595-602.

32. De Swert KO, Joos GF. Extending the understanding of sensory neuropeptides. Eur Pharmacol. 2006;533(1-3):171-81.

33. 33. Forsyth RD, Cole P, Shephard RJ. Exercise and nasal patency. J Appl Physiol Respir Environ Exerc Physiol. 1983;55(3):8605

34. 34. Bougault V, Loubaki L, Joubert P, Turmel J, Couture C, Laviolette M, et al. Airway remodeling and inflammation in competitive swimmers training in indoor chlorinated swimming pools. J Allergy Clin Immunol. 2012;129(2):351-8, 8 e 1.

35. Carbonnelle S, Francaux M, Doyle I, Dumont $X$, de Burbure C, Morel G, et al. Changes in serum pneumoproteins caused by shortterm exposures to nitrogen trichloride in indoor chlorinated swimming pools. Biomarkers. 2002;7(6):464-78.

36. Seys SF, Feyen L, Keirsbilck S, Adams E, Dupont LJ, Nemery B. An outbreak of swimming-pool related respiratory symptoms: An elusive source of trichloramine in a municipal indoor swimming pool. Int J Hyg Environ Health. 2015;218(4):386-91.

\section{Dr. Brecht Steelant}

Clinical Immunology

Department of Microbiology and

Immunology

KU Leuven

Herestraat 49, box 811

3000 Leuven

Belgium

Tel.: +32 16346165

Fax: +32 16343835

E-mail: brecht.steelant@kuleuven.be 
A

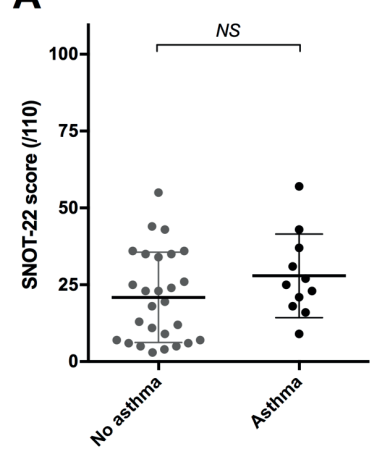

D

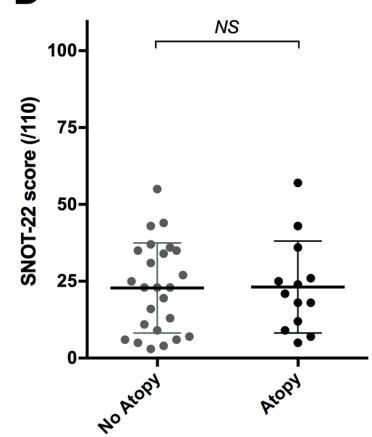

G

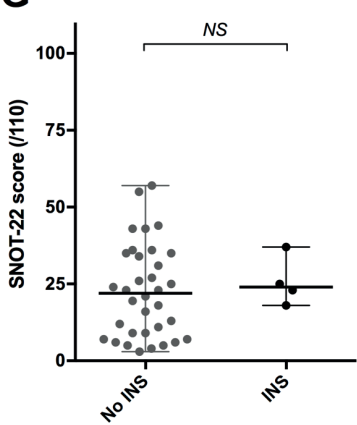

J

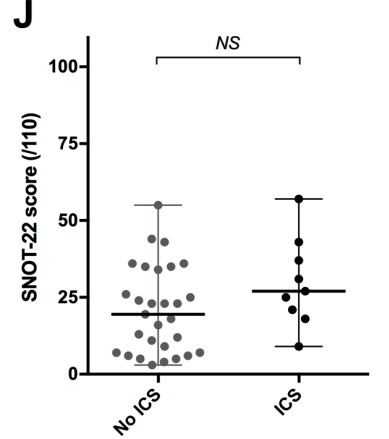

B

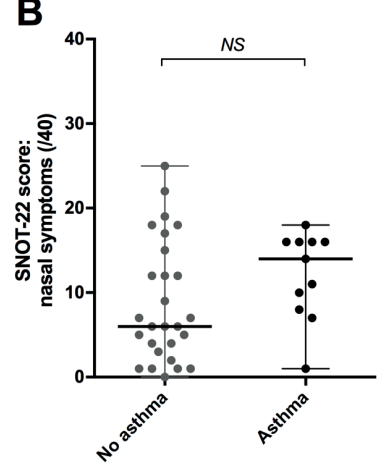

E

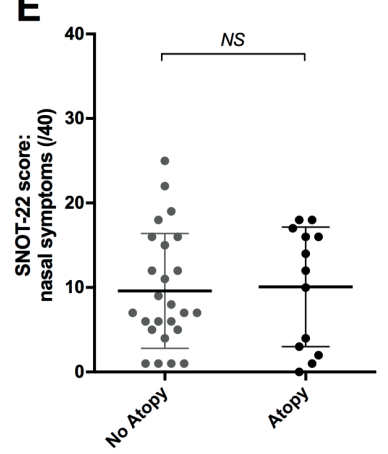

H
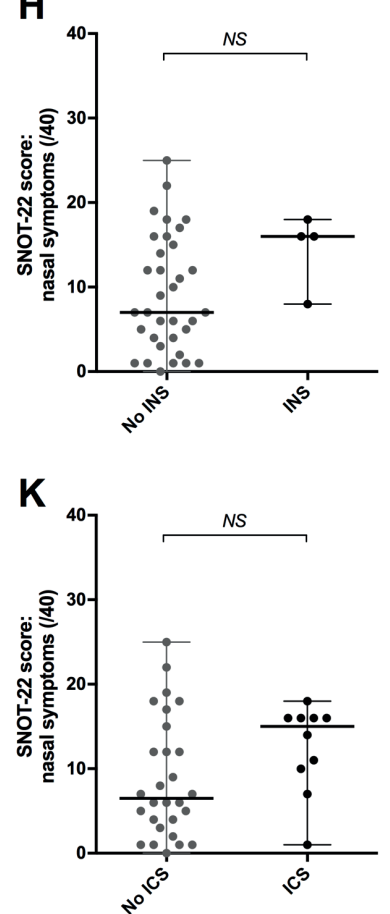

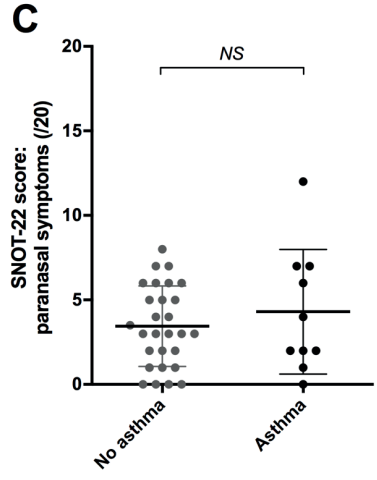

$\mathbf{F}$

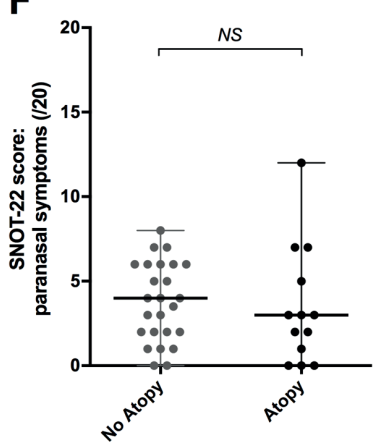

I

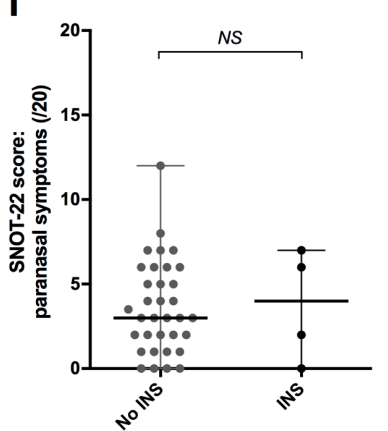

L

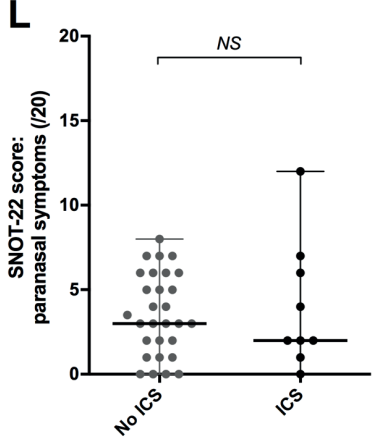

Figure E1. Asthma, atopy, usage of inhalation corticosteroids (ICS) or intranasal steroids (INS) did not influence SNOT-scores in elite swimmers. 